\title{
A Peptide-Based Enzyme-Linked Immunosorbent Assay for Detecting Antibodies Against Avian Infectious Bronchitis Virus
}

\author{
Liping Yin ${ }^{1,2+}$, Qi Wu ${ }^{1,3+}$, Zhixian Lin ${ }^{1,3}$, Kun Qian ${ }^{1,3,4}$, Hongxia Shao ${ }^{1,3,4}$, Zhimin Wan ${ }^{1,3,4}$, \\ Yuelong Liu ${ }^{1,2}$, Jianqiang Ye ${ }^{1,3,4 *}$ and Aijian Qin ${ }^{1,3,4 *}$ \\ 1 Key Laboratory of Avian Preventive Medicine, Ministry of Education, Yangzhou University, Yangzhou, China, ${ }^{2}$ JiangsuLihua \\ Animal Husbandry Co., Ltd, Changzhou, China, ${ }^{3}$ Jiangsu Co-innovation Center for Prevention and Control of Important \\ Animal Infectious Diseases and Zoonoses, Yangzhou, China, ${ }^{4}$ The International Joint Laboratory for Cooperation in \\ Agriculture and Agricultural Product Safety, Ministry of Education, Yangzhou University, Yangzhou, China
}

OPEN ACCESS

Edited by:

Constantinos S. Kyriakis, Auburn University, United States

Reviewed by:

Vicky Lin van Santen, Auburn University, United States Ahmed Ali,

Beni-Suef University, Egypt

*Correspondence: Jianqiang Ye jqye@yzu.edu.cn

Aijian Qin

aijian@yzu.edu.cn

tThese authors have contributed equally to this work

Specialty section:

This article was submitted to Veterinary Infectious Diseases, a section of the journal Frontiers in Veterinary Science

Received: 20 October 2020 Accepted: 16 December 2020

Published: 21 January 2021

Citation: Yin L, Wu Q, Lin Z, Qian K, Shao H, Wan Z, Liu Y, Ye J and Qin A (2021) A

Peptide-Based Enzyme-Linked Immunosorbent Assay for Detecting Antibodies Against Avian Infectious

Bronchitis Virus.

Front. Vet. Sci. 7:619601.

doi: 10.3389/fvets.2020.619601
Infectious bronchitis virus (IBV) causes substantial loss to the poultry industry despite extensive vaccination. Assessing the antibody response is important for the development and evaluation of effective vaccines. We have developed an enzyme-linked immunosorbent assay (ELISA) for the detection of IBV-specific antibodies, using a synthetic peptide based on a conserved sequence in the IBV spike protein. This peptide-based ELISA (pELISA) specifically detects antibodies to different genotypes of IBV but not antibodies against other common chicken viruses. This assay could detect IBV-specific antibody response on as early as day 7 postinfection. In the testing with field serum samples collected from chickens administered with IBV vaccines, the sensitivity, specificity, and accuracy of pELISA were $98.30,94.12$, and $98.8 \%$, respectively, relative to indirect immunofluorescence assay. Our data demonstrate that the pELISA is of value for the detection of IBV antibody and the evaluation of IBV vaccines.

Keywords: infectious bronchitis virus, pELISA, antibody, detection, chicken

\section{INTRODUCTION}

Avian infectious bronchitis, a highly contagious disease, is caused by a coronavirus, that is, infectious bronchitis virus (IBV). The infection of IBV generally causes serious respiratory and renal diseases in broilers and lowers egg production in layers (1), resulting in significant economic loss in the poultry industry (2). Although the efficacy is far from optimal, vaccines represent one of the most effective tools for the control of IBV. As for other animal and human vaccines, assessment of antibody response is of key importance for IBV vaccine development.

The IBV genome encodes four structural proteins as well as at least 15 non-structural and accessory proteins (1). Among these proteins, the surface spike (S) glycoprotein is the major antigen that induces protective immune response against IBV (3). The S protein consists of two subunits, S1 and S2, with the S1 subunit being responsible for binding cellular receptors (4) and the major target of neutralizing antibodies. The S2 subunit is more conserved than $\mathrm{S} 1$ and also plays a role in inducing protective immune response (5-7), as well as facilitating membrane fusion and viral entry $(5,8,9)$. It has been reported that $S 2$ could produce cross-protection against strains that differ in their S1 subunits (7).

A feasible and practical immunoassay for antibody detection and immune response measurement is critical for vaccine development. Enzyme-linked immunosorbent assays (ELISAs) 
based on whole IBV viral particles, as well as recombinant S1, nucleocapsid, and non-structural proteins, have been reported for detecting antibodies against IBV (10-13). Although these assays have achieved promising results, they have some limitations, especially in detecting antibodies induced by emergent or variant IBV strains.

Our previous studies revealed an epitope in S2 and identified the key amino acids in this epitope (14). Based on this finding, we have designed an IBV S2-based peptide and developed an ELISA for the detection of antibodies against IBV.

\section{MATERIALS AND METHODS}

\section{Synthetic Peptide and Serum Samples}

A 20-mer peptide, SCPYVSYGRFCIQPDGSIKQ, corresponding to amino acid positions 8 to 27 on the S2 protein of IBV CK/CH/2010/JT1 strain (GenBank KU361187), was synthesized
(Synpeptide Co., Ltd., Shanghai, China) and used as the coating antigen for the peptide-based ELISA (pELISA). Serum samples that were used in our study included 100 serum samples collected from specific-pathogen-free (SPF) chickens (Spirax Ferrer Poultry Science and Technology Co., Ltd., Jinan, China), 250 serum samples collected from chickens that were vaccinated with IBV vaccines $\mathrm{H} 120$ and H52 (Lihua Animal Husbandry Co., LTD, Jiangsu, China), and sera against IBV strains Massachusetts 41 (M41), 4/91, H52, H120, and $\mathrm{CK} / \mathrm{CH} / 2010 / \mathrm{JT} 1$, which were prepared in our laboratory by infecting SPF chickens with 1,000 median egg infectious dose $\left(E_{50}\right)$ of each strain. Immune serum against QXL87 (GenBank accession no. MH743141) vaccine strain (QX-type) was obtained from Zhongchong Sino Biological Technology Co., Ltd. (Shanghai China). The other sera were kept in our laboratory, which were made from SPF chickens infected with the viruses (15).

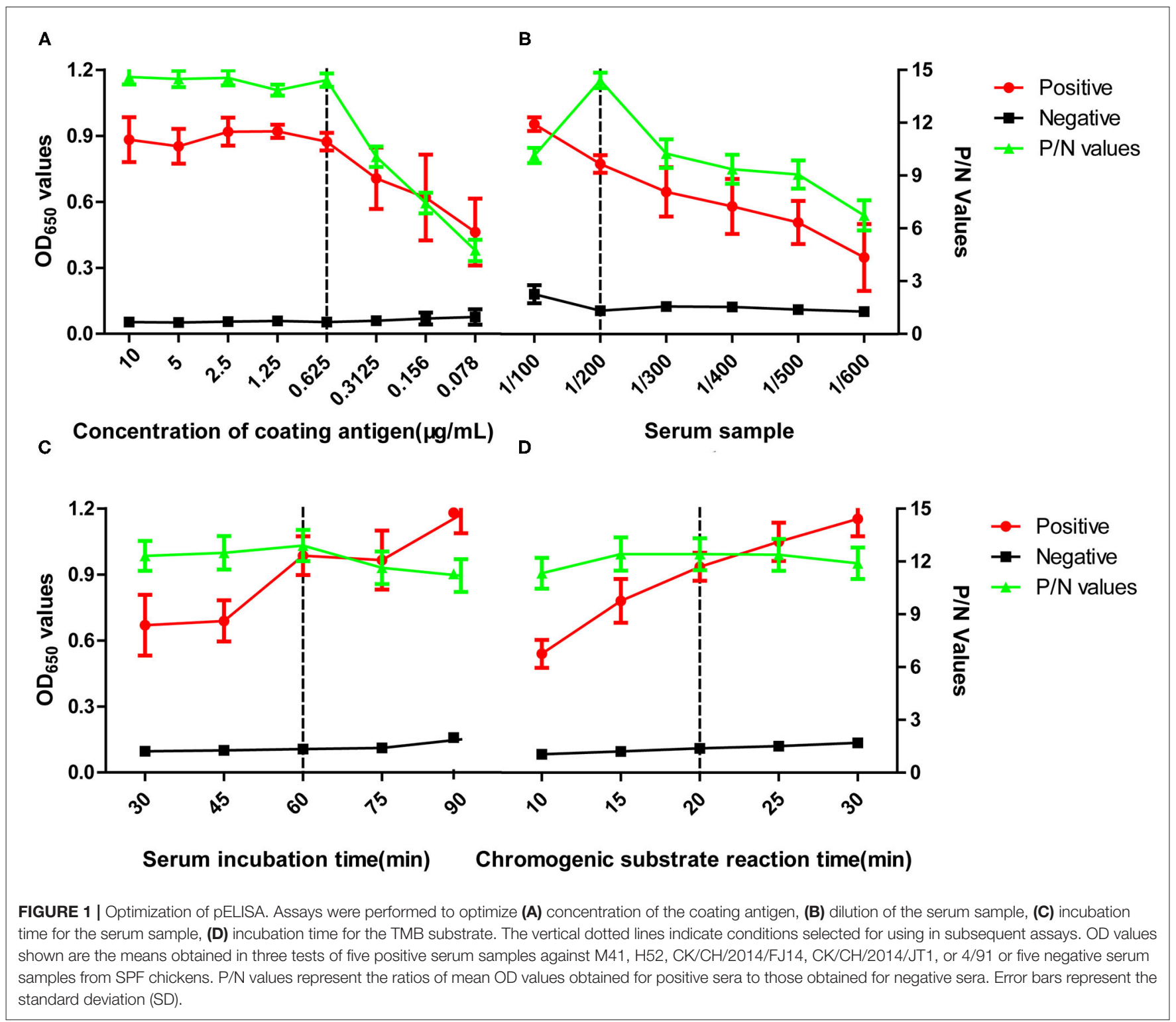




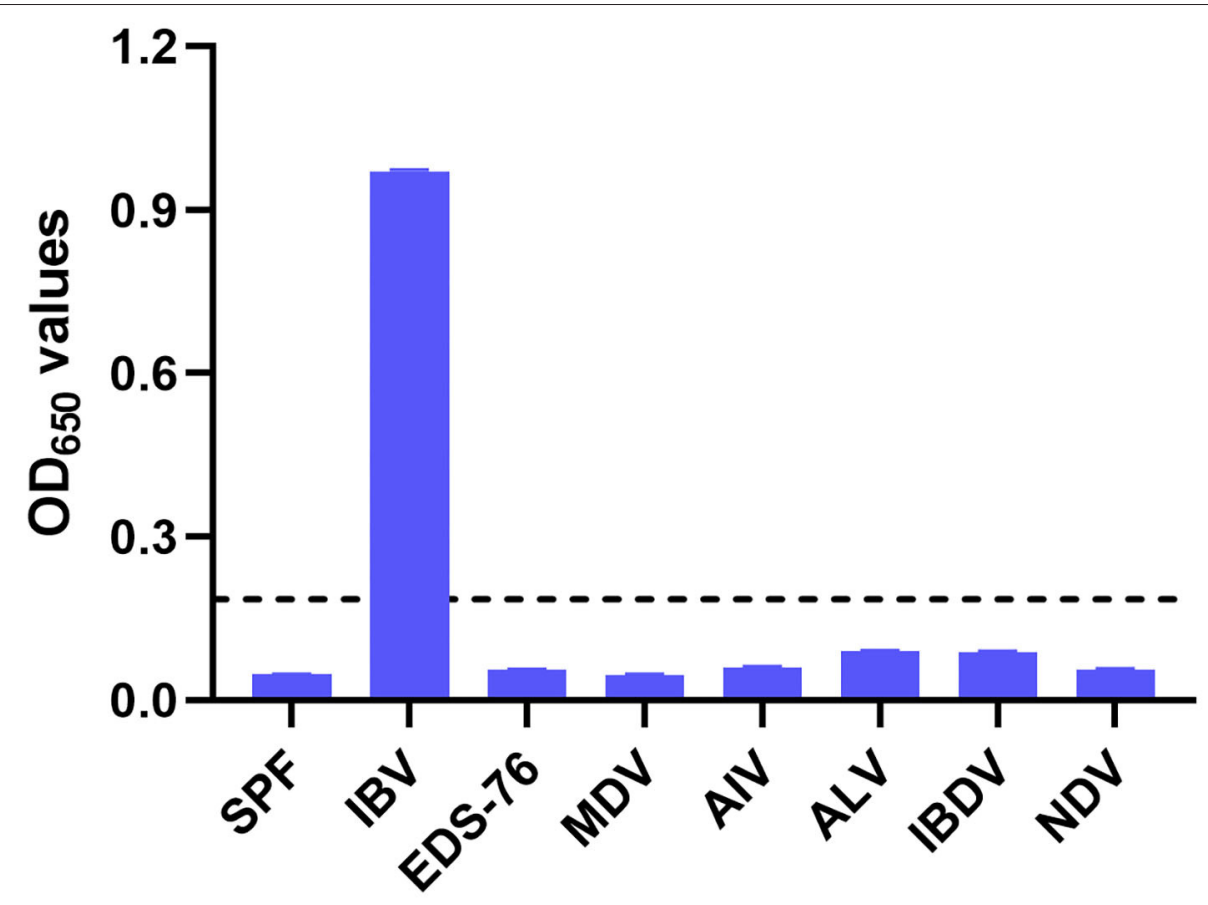

FIGURE 2 | Specificity of the pELISA. Specificity of the pELISA in detecting antibodies against common chicken viruses. Antisera raised against Newcastle disease virus (NDV), avian influenza virus (AIV, H9N2), avian leukosis virus (ALV), Marek's disease virus (MDV), egg drop syndrome virus (EDS-76V), and infectious bursal disease virus (IBDV) were tested by pELISA. Their $\mathrm{OD}_{650}$ values were lower than the cutoff value. The black horizontal dotted line indicates the cutoff.

\section{pELISA Procedure}

For the pELISA, 96-well polystyrene plates were coated with $0.63 \mu \mathrm{g} / \mathrm{ml}$ of the synthetic peptide in $0.1 \mathrm{M}$ carbonate buffer $(\mathrm{pH}$ 9.6) at $4^{\circ} \mathrm{C}$ overnight. After washing with phosphate-buffered saline containing $0.05 \% \mathrm{vol} / \mathrm{vol}$ Tween 20 (PBST), the plates were blocked with $300 \mu \mathrm{l} /$ well of $8 \%$ rabbit serum in PBST (Lanzhou Minhai Biological Engineering Co., Ltd., China) for $3 \mathrm{~h}$ at $37^{\circ} \mathrm{C}$. Following three washes with PBST, $100 \mu \mathrm{l}$ serum (1:200 dilution in PBST) was added to the wells, and the plates were incubated at $37^{\circ} \mathrm{C}$ for $1 \mathrm{~h}$. The plates were then washed five times with PBST and further incubated with $100 \mu \mathrm{l} /$ well horseradish peroxidase-conjugated goat anti-chicken immunoglobulin G (IgG) $(1: 20,000)$ (Jackson ImmunoResearch Laboratories, Inc., USA) for $1 \mathrm{~h}$ at $37^{\circ} \mathrm{C}$. After washing for five times, the signals were developed by incubating the plates with $100 \mu \mathrm{L} /$ well TMB substrate for $20 \mathrm{~min}$ at $37^{\circ} \mathrm{C}$, followed by stopping the color development with $100 \mu \mathrm{l} /$ well of $1 \%$ sodium dodecyl sulfate. $\mathrm{OD}_{650}$ values were read with an ELISA reader (BioTek, VT, USA). Each assay was repeated twice.

For the commercial IBV antibody ELISA kit (IDEXX, Westbrook, ME), the assay was performed according to the manufacturer's protocol. Briefly, $100 \mu \mathrm{l}$ of 1:500 diluted sera was added to each well. After incubation for $30 \mathrm{~min}$ at room temperature and washing for three times, $100 \mu \mathrm{l}$ conjugated antibody was added and incubated for $30 \mathrm{~min}$ at room temperature. The plates were washed five times, and the substrate was added in the well for color development for $15 \mathrm{~min}$ and terminated with stop solution. $\mathrm{OD}_{650}$ values were read with an ELISA reader (BioTek, VT, USA).
TABLE 1 | Reproducibility of the pELISA in detecting IBV-specific antibodies.

\begin{tabular}{lccccc}
\hline Samples & \multicolumn{2}{c}{ Intra-assay Variability } & & \multicolumn{2}{c}{ Interassay Variability } \\
\cline { 2 - 3 } \cline { 6 - 6 } & Mean \pm SD & CV (\%) & & Mean \pm SD & CV (\%) \\
\hline 1 & $0.043 \pm 0.001$ & $2.3 \%$ & & $0.048 \pm 0.001$ & $2.7 \%$ \\
2 & $0.046 \pm 0.001$ & $1.6 \%$ & & $0.046 \pm 0.001$ & $1.9 \%$ \\
3 & $0.049 \pm 0.001$ & $2.3 \%$ & & $0.051 \pm 0.001$ & $1.7 \%$ \\
4 & $0.046 \pm 0.001$ & $1.6 \%$ & & $0.045 \pm 0.001$ & $2.5 \%$ \\
5 & $0.842 \pm 0.016$ & $1.9 \%$ & & $0.817 \pm 0.038$ & $4.7 \%$ \\
6 & $0.884 \pm 0.009$ & $1.8 \%$ & & $0.433 \pm 0.025$ & $6.5 \%$ \\
7 & $0.421 \pm 0.006$ & $2.4 \%$ & & $0.249 \pm 0.003$ & $1.2 \%$ \\
8 & $0.491 \pm 0.008$ & $4.4 \%$ & & $0.198 \pm 0.002$ & $1.3 \%$ \\
\hline
\end{tabular}

Every sample repeated three times, SD, standard deviation; $C V$, coefficient of variation.

\section{Cutoff Value, Specificity, and Reproducibility}

After the optimal dilution and peptide concentration were set, 100 negative serum samples from SPF chickens were tested by pELISA to set a cutoff value. To examine the specificity of the pELISA, positive serum samples against avian influenza virus (AIV), avian leukemia virus (ALV), Newcastle disease virus (NDV), infectious bursal disease virus (IBDV), Marek's disease virus (MDV), and egg drop syndrome virus (EDS-76V) were tested. Evaluation of the assay reproducibility within and between runs was performed with eight serum samples, four positive and another four negative, which were confirmed in indirect 
immunofluorescence assay (IFA). For intra-assay (within-plate) reproducibility, four replicates of each serum sample were analyzed within the same plate, and the experiment was performed three times independently. For interassay (betweenrun) reproducibility, four replicates of each serum sample were run in different plates. This test was performed three times using plates coated at different times. The mean $\mathrm{OD}_{650}$ value, standard deviation (SD), and coefficient of variation (CV) were calculated.

\section{Performance of the pELISA}

To further evaluate the pELISA, 250 serum samples from vaccinated chickens were tested by pELISA, IFA, and the commercial ELISA kit (IDEXX, USA). The IFA was conducted according to the previously reported protocol (16). Briefly, primary chicken embryo kidney cells were grown in the 96-well plates and infected with IBV M41 strain. After 2 days, the cells were fixed with acetone and alcohol (3:2) for $5 \mathrm{~min}$. The sera diluted 1:200 with PBS were added to the wells and incubated for $60 \mathrm{~min}$. After washing with PBST, cells were incubated with the fluorescein isothiocyanate-conjugated rabbit-anti-chicken IgG for $60 \mathrm{~min}$, followed by washing five times and observation under the fluorescence microscope. The sensitivity, specificity, and the accuracy of the pELISA and commercial ELISA kit were evaluated by comparing to the data generated by IFA.

To further evaluate the assay, five 6-week-old SPF chickens were vaccinated intranasally with $10^{3}$ EID $_{50}$ IBV strain $\mathrm{H} 52$ or
4/91; sera were collected on days $3,7,14,21$, and 28 and tested by the pELISA and the commercial IDEXX ELISA kit.

\section{RESULTS}

\section{Optimization of the pELISA}

To optimize the pELISA, two-fold serial dilutions of the synthetic peptide and different dilutions of the positive and negative chicken serum samples, which had been confirmed by IFA, were tested. As shown in Figure 1, the optimal concentration of peptide was found to be $0.63 \mu \mathrm{g} / \mathrm{ml}$, and the dilution of serum samples was 1:200 (Figure 1), based on the criteria that the $\mathrm{P} / \mathrm{N}$ value of the $\mathrm{OD}_{650}$ ratio between positive and negative sera was highest. At the same time, the best results could reach at 60-min incubation for serum samples and second conjugated antibody (Figure 1).

\section{Cutoff Value, Specificity, and Reproducibility of the pELISA}

The 100 negative serum samples collected from SPF chickens were tested to set up a cutoff value for the pELISA. When tested at a 1:200 dilution, these samples gave a mean $\mathrm{OD}_{650}$ value of 0.067 , with an SD of 0.017 ; thus, the cutoff value was defined as 0.185 ( 2 mean $\pm 3 \mathrm{SD}$ ). In the subsequent assays, serum samples giving $\mathrm{OD}_{650}$ values $\geq 0.185$ were designated positive for IBV antibodies, whereas those generating $\mathrm{OD}_{650}$ values $<0.185$

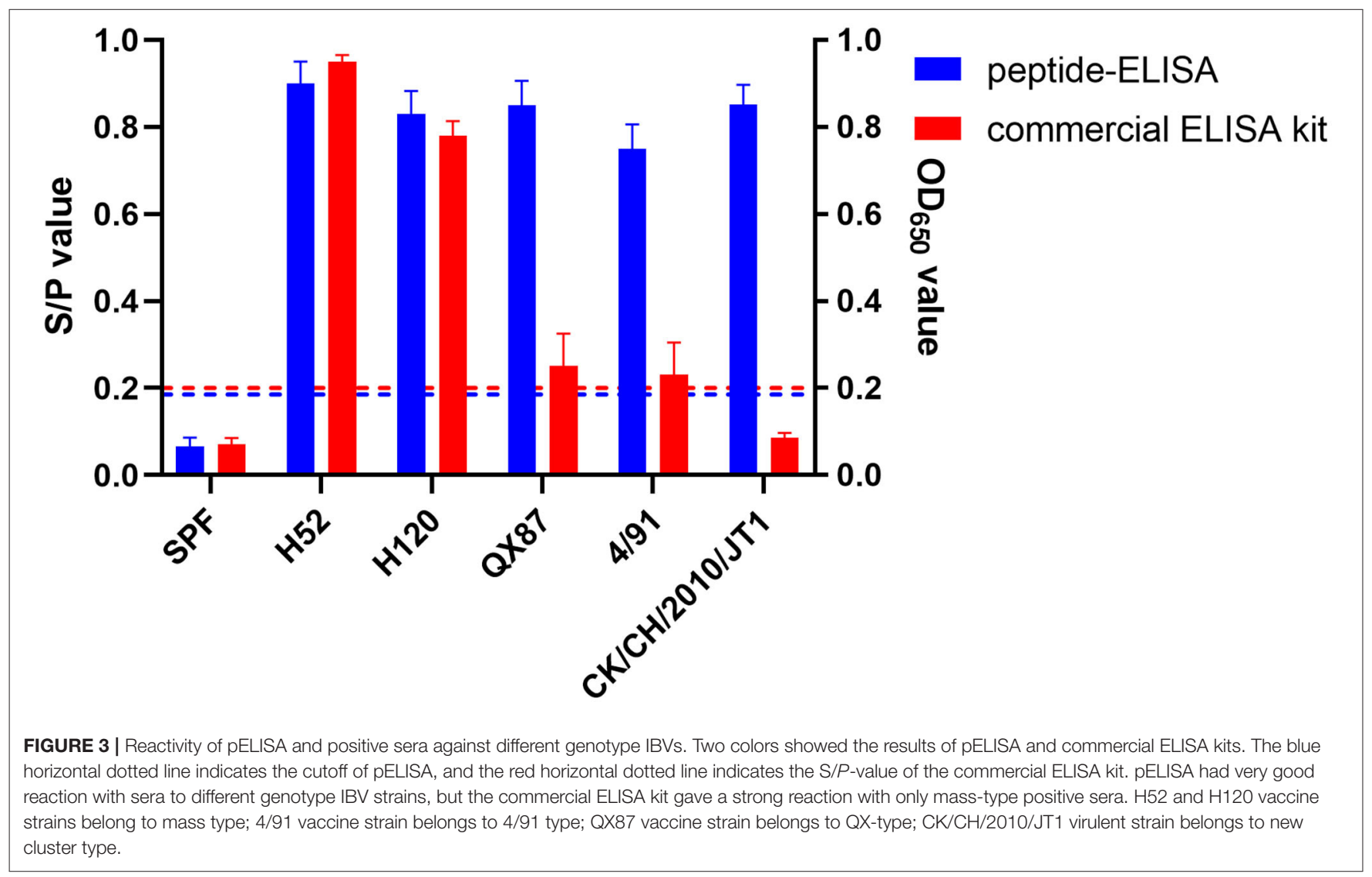


were designated negative. The specificity of the pELISA was evaluated by testing the reactivity of sera raised against NDV, ALV, IBDV, AIV, MDV, and EDS-76V. As shown in Figure 2, no cross-reaction between the IBV S2 peptide antigen and these sera was detected, demonstrating the specificity of the pELISA.

Next, we examined the reproducibility of the assay. Eight selected serum samples, four positive and four negative, were tested by the pELISA in quadruplicate. The interassay $\mathrm{CV}$ was 1.2 to $6.5 \%$, and the intra-assay CV ranged from 1.6 to $4.4 \%$ (Table 1), indicating the high reproducibility of our assay.

\section{pELISA Detects Antibodies Against Different Genotypes of IBV}

To evaluate whether our pELISA is suitable for detecting antibodies against various genotypes of IBV, chicken sera raised against IBVs 4/91, H52, H120, QX87 (QX type), and $\mathrm{CK} / \mathrm{CH} / 2010 / \mathrm{JT} 1$ (New cluster type) were tested in the assay. The pELISA could detect antibodies against all of these different IBVs. Interestingly, the commercial IDEXX ELISA kit could detect sera raised against the $\mathrm{H} 52$ and $\mathrm{H} 120$ strains, but showed very weak reaction with the sera to 4/91, QX87, or CK/CH/2010/JT1 (Figure 3), which indicated that the pELISA is a better option for the detection of antibodies to various IBV genotypes.

To further evaluate the sensitivity, specificity, and accuracy of pELISA, 250 serum samples from chickens immunized with H120 and H52 vaccines were tested with the pELISA, IFA, and the commercial ELISA kit. As shown in Table 2, 232 and 18 of these serum samples tested positive and negative by pELISA, respectively, whereas 233 and 17 tested positive and negative by IFA, respectively. Compared to the results of IFA, the sensitivity, specificity, and the accuracy of the PELISA were 99.14, 94.12, and $98.80 \%$, respectively. With the commercial kit, 230 and 20 of these 250 serum samples were positive and negative, respectively. Compared to the results of IFA, the sensitivity, specificity, and the accuracy of the commercial kit were 96.57, 70.59, and $94.80 \%$, respectively.

\section{pELISA Effectively Detects Antibody Response in Chickens}

Finally, we evaluated the suitability of the pELISA in measuring the immune response elicited by the IBV vaccine. Chickens were vaccinated with IBV H52 and 4/91 vaccines, and sera were collected at different time points postvaccination and tested by pELISA. In sera from H52-vaccinated chickens, the pELISA was able to detect antibody response as early as on day 7 postvaccination, with three of the six chickens being positive, and all chickens were positive by 14, 21, and 28 days postvaccination, whereas the commercial ELISA kit could detect positive antibodies on day 14 postvaccination. In sera from 4/91 vaccinated chickens, the pELISA was able to detect antibody response as early as on day 7 postvaccination, with two of the six chickens being positive, and all chickens were positive by 14 , 21, and 28 days postvaccination. By contrast, the earliest time point when the commercial ELISA kit could detect a positive antibody response was on day 14 postvaccination, whereas no positive antibody response was detected in any of the birds on
TABLE 2 | Comparison of the pELISA, IFA, and the commercial IBV ELISA kit for 250 field serum samples.

\begin{tabular}{|c|c|c|c|}
\hline $\begin{array}{l}\text { Serum } \\
\text { samples }\end{array}$ & IFA & pELISA & IDEXX \\
\hline 223 serum samples ${ }^{a}$ & + & + & + \\
\hline 8,283 & + & + & - \\
\hline 8,284 & + & + & - \\
\hline 6,817 & + & + & - \\
\hline 6,823 & + & + & - \\
\hline 6,878 & + & + & - \\
\hline 6,872 & + & + & - \\
\hline 6,819 & + & + & - \\
\hline 8,354 & + & + & - \\
\hline 86 & - & + & - \\
\hline 23 & + & - & + \\
\hline 8,358 & + & - & + \\
\hline 8,342 & - & - & + \\
\hline 8,348 & - & - & + \\
\hline 8,370 & - & - & + \\
\hline 8,366 & - & - & + \\
\hline 8,371 & - & - & + \\
\hline 11 serum samples ${ }^{b}$ & - & - & - \\
\hline
\end{tabular}

+, Positive; -, negative.

${ }^{a}$ Number of serum samples tested positive by all three methods.

${ }^{b}$ The number of the negative serum samples by three methods.

TABLE 3 | Comparison of pELISA and a commercial ELISA kit in detecting IBV antibody response in chickens ${ }^{\mathrm{a}}$.

\begin{tabular}{llccccc}
\hline & & Day 3 & Day 7 & Day 14 & Day 21 & Day 28 \\
\hline H52 & pELISA & 0/6 & $3 / 6$ & $6 / 6$ & $6 / 6$ & $6 / 6$ \\
& Commercial & $0 / 6$ & $0 / 6$ & $6 / 6$ & $6 / 6$ & $6 / 6$ \\
& ELISA kit & & & & & \\
\multirow{2}{*}{$4 / 91$} & pELISA & $0 / 6$ & $2 / 6$ & $6 / 6$ & $6 / 6$ & $6 / 6$ \\
& Commercial & $0 / 6$ & $0 / 6$ & $2 / 6$ & $4 / 6$ & $5 / 6$ \\
& ELISA kit & & & & & \\
\hline
\end{tabular}

${ }^{a}$ Chickens were vaccinated with IBV H52 and 4/91, and sera were collected on indicated days and tested by PELISA and the IDEXX ELISA kit.

${ }^{b}$ The number are positive number/total number.

day 7 postinfection. Further, on days 14, 21, and 28, only two four and five of the chickens tested positive by the IDEXX kit, respectively (Table 3 ).

\section{DISCUSSION}

ELISA has been used in IBV serological tests for its feasibility, sensitivity, rapidity and being suitable for large-scale use (17). In the previously reported IBV antibody detection assays, whole IBV virions and recombinant proteins were used as antigens (1013). Although these assays have their advantages, preparation of the antigens was time-consuming and expensive. In this study, we developed a pELISA for IBV antibody detection, using a peptide based on the S2 sequence. In contrast to S1, which 
is highly variable among different IBV strains, S2 is highly conserved and carries conserved epitopes $(7,14,18)$. Our results demonstrated that the pELISA could detect antibodies against different genotypes of IBV. Furthermore, the assay is highly sensitive, specific, and accurate compared with the results of IFA and the commercial ELISA kit, highlighting its value as a reliable assay for the detection of IBV antibodies.

For antibody detection, different methods could give different results. The same method with different antigens also gives different results $(10,19)$. In our pELISA, we could detect antibody against IBV on as early as 7 day postinfection. However, the commercial ELISA kit could not detect the antibody until 14 days postvaccination, and the positive value was not very strong. Our results are similar to those of Kutle et al. (20), who found that the titer of antibody to IBV was very low on 20 days postvaccination when they used the commercial ELISA kit. The reasons could be mainly because of the surface antigen variation of the virions and different coated protein. In addition, high concentration of epitope of S2 protein could have good reaction with the antibody to the conserved epitope in different genotype. It indicates that the epitope in S2 could be an important antigen in IBV immune response.

In summary, we have developed a pELISA with a synthetic S2 peptide, which could detect antibodies against different genotypes of IBV. This assay possesses sufficient sensitivity, specificity, and accuracy and has the potential to serve as a rapid and reliable method for IBV antibody detection.

\section{REFERENCES}

1. Cavanagh D. Coronavirus avian infectious bronchitis virus. Vet Res. (2007) 38:281-97. doi: 10.1051/vetres:2006055

2. Jackwood MW. Review of infectious bronchitis virus around the world. Avian Dis. (2012) 56:634-41. doi: 10.1637/10227-043012Review.1

3. Ignjatovic J, Galli L. The S1 glycoprotein but not the $\mathrm{N}$ or $\mathrm{M}$ proteins of avian infectious bronchitis virus induces protection in vaccinated chickens. Arch Virol. (1994) 138:117-34. doi: 10.1007/BF013 10043

4. Belouzard S, Millet JK, Licitra B N, Whittaker G R. Mechanisms of coronavirus cell entry mediated by the viral spike protein. Viruses. (2012) 4:1011-33. doi: 10.3390/v4061011

5. Bickerton E, Maier HJ, Stevenson-Leggett P, Armesto M, Britton P. The S2 subunit of infectious bronchitis virus beaudette is a determinant of cellular tropism. J Virol. (2018) 92:e01044-18. doi: 10.1128/JVI.01044-18

6. Koch G, Hartog L, Kant A, van Roozelaar DJ. Antigenic domains on the peplomer protein of avian infectious bronchitis virus: correlation with biological functions. J Gen Virol. (1990) 71 (Pt 9):1929-35. doi: 10.1099/0022-1317-71-9-1929

7. Toro H, Zhao W, Breedlove C, Zhang Z, Yu Q, Van Santen V. Infectious bronchitis virus $\mathrm{S} 2$ expressed from recombinant virus confers broad protection against challenge. Avian Dis. (2014) 58:83-9. doi: 10.1637/10641-081613-Reg.1

8. Bickerton E, Dowgier G, Britton P. Recombinant infectious bronchitis viruses expressing heterologous S1 subunits: potential for a new generation of vaccines that replicate in Vero cells. J Gen Virol. (2018) 99:1681-5. doi: 10.1099/jgv.0.001167

9. Cheng J, Zhao Y, Xu G, Zhang K, Jia W, Sun Y, et al. The S2 subunit of QX-type infectious bronchitis coronavirus spike protein is an essential determinant of neurotropism. Viruses. (2019) 11:972. doi: 10.3390/v11100972

\section{DATA AVAILABILITY STATEMENT}

The original contributions generated for the study are publicly available. This data can be found at: https://www.ncbi.nlm.nih. gov/nuccore/KU361187.1/.

\section{ETHICS STATEMENT}

The animal study was reviewed and approved by Animal Care and ethics Committee at Yangzhou University.

\section{AUTHOR CONTRIBUTIONS}

This manuscript was written by LY and AQ. Experiment and data analysis were performed by LY, QW, ZL, and YL. Study designed by ZW, AQ, JY, KQ, and HS. All authors contributed to the article and approved the submitted version.

\section{FUNDING}

This study was supported by the National Key Research and Development Program of China (2016YFD0500803), the Priority Academic Program Development of Jiangsu Higher Education Institutions and the Jiangsu Co-innovation Center for the Prevention and Control of Important Animal Infectious Diseases and Zoonoses.

10. Gibertoni AM, Montassier MDS, Sena JAD, Givisiez PEN, Furuyama CRAG, Montassier HJ. Development and application of a Saccharomyces cerevisiaeexpressed nucleocapsid protein-based enzyme-linked immunosorbent assay for detection of antibodies against infectious bronchitis virus. J Clin Microbiol. (2005) 43:1982-4. doi: 10.1128/JCM.43.4.1982-19 84.2005

11. Lei J, Shi T, Sun D, Mo K, Yan Y, Jin Y, et al. Development and application of nsp5-ELISA for the detection of antibody to infectious bronchitis virus. J Virol Methods. (2017) 243:182-9. doi: 10.1016/j.jviromet.2017. 01.026

12. Marquardt WW, Snyder DB, Schlotthober BA. Detection and quantification of antibodies to infectious bronchitis virus by enzyme-linked immunosorbent assay. Avian Dis. (1981) 25:713-22.

13. Wang $\mathrm{CH}$, Hong $\mathrm{CC}$, Seak JCH. An ELISA for antibodies against infectious bronchitis virus using an S1 spike polypeptide. Vet Microbiol. (2002) 85:333-42. doi: 10.1016/s0378-1135(01) 00525-9

14. Wu Q, Lin ZX, Qian K, Shao HX, Ye JQ, Qin AJ. Peptides with $16 \mathrm{R}$ in $\mathrm{S} 2$ protein showed broad reactions with sera against different types of infectious bronchitis viruses. Vet Microbiol. (2019) 236:108391. doi: 10.1016/j.vetmic.2019.108391

15. Zhou H, Zhang M, Tian X, Shao H, Qian K, Ye J, et al. Identification of a novel recombinant virulent avian infectious bronchitis virus. Vet Microbiol. (2017) 199:120-127. doi: 10.1016/j.vetmic.2016. 12.038

16. Wang P, Zhang J, Wang W, Li T, Liang G, Shao H, et al. A novel monoclonal antibody efficiently blocks the infection of serotype 4 fowl adenovirus by targeting fiber-2. Vet Res. (2018) 49:29. doi: 10.1186/s13567-0180525-y

17. Villarreal LYB. Diagnosis of infectious bronchitis: an overview of concepts and tools. Braz J Poultry Sci. (2010) 12:111-4. doi: 10.1590/S1516-635X2010000200006 
18. Ignjatovic J, Sapats S. Identification of previously unknown antigenic epitopes on the $\mathrm{S}$ and $\mathrm{N}$ proteins of avian infectious bronchitis virus. Arch Virol. (2005) 150:1813-31. doi: 10.1007/s00705-005-0541-x

19. Chen HY, Coote B, Attree S, Hiscox JA. Evaluation of a nucleoprotein-based enzyme-linked immunosorbent assay for the detection of antibodies against infectious bronchitis virus. Avian Pathol. (2003) 32:519-26. doi: 10.1080/0307945031000 154125

20. Kutle L, Ljuma Skupnjak L, Vrdoljak A, Jankovic D, Boelm GJ, Kelemen F, et al. Efficacy of infectious bronchitis GI-13 (793B) vaccine candidate tested according to the current european union requirements and for cross-protection against heterologous QX-like challenge. Viral Immunol. (2020) 33:555-64. doi: 10.1089/vim.2020. 0011
Conflict of Interest: LY and YL were employed by the company JiangsuLihua Animal Husbandry Co., Ltd.

The remaining authors declare that the research was conducted in the absence of any commercial or financial relationships that could be construed as a potential conflict of interest.

Copyright (C) 2021 Yin, Wu, Lin, Qian, Shao, Wan, Liu, Ye and Qin. This is an open-access article distributed under the terms of the Creative Commons Attribution License (CC BY). The use, distribution or reproduction in other forums is permitted, provided the original author(s) and the copyright owner(s) are credited and that the original publication in this journal is cited, in accordance with accepted academic practice. No use, distribution or reproduction is permitted which does not comply with these terms. 